\title{
Direct Numerical Simulation of the Bending Effect in Turbulent Premixed Flames
}

\author{
Girish V. Nivarti ${ }^{\mathrm{a}, *}$, R. Stewart Cant ${ }^{\mathrm{a}}$ \\ ${ }^{a}$ University Engineering Department, Trumpington Street \\ Cambridge CB2 1PZ, United Kingdom
}

\begin{abstract}
In turbulent premixed flames, much experimental evidence points to a strong influence of pre-mixture turbulence intensity on the turbulent burning velocity. The linear enhancement of turbulent burning velocity in low-intensity turbulence is predicted accurately by current models. In contrast, the deviation from linearity in high-intensity turbulence, known as the "bending effect," remains to be explained. The present work has employed Direct Numerical Simulation (DNS) to investigate the bending effect. An initially laminar methane-air premixed flame was subjected to increasing levels of turbulence across five different simulations which maintained all parameters except the turbulence intensity constant. The bending effect was captured within these simulations. Subsequently, plausible explanations were investigated using the framework of the Flame Surface Density (FSD) approach. From the ensuing analysis, it is evident that flame surface area reflects distinctly the variation of turbulent burning velocity with turbulence intensity. Local flame quenching does not appear to be the primary mechanism behind the bending effect. Instead, the observed bending effect results from a shift in balance, under high-intensity turbulence, towards mechanisms that favour destruction of flame surface area. These mechanisms tend to preserve the reaction layer and, thereby, ensure the validity of Damköhler's hypothesis and flamelet models in conditions that cause the bending effect that is observed here to occur.
\end{abstract}

Keywords: Turbulent premixed flames, the bending effect, Direct Numerical Simulation, high-intensity turbulence.

\section{Motivation}

Turbulent burning velocity $s_{T}$ is a basic measure of how fast a turbulent fuel-air mixture burns. It is defined using the global transformation rate of reactants to products through the turbulent premixed flame brush:

$$
s_{T} \equiv-\frac{1}{\rho_{u} Y_{u, F} A_{0}} \int_{V} \dot{\omega}_{F} d V,
$$

where $\rho_{u}$ is the density of unburned gas, $Y_{u, F}$ is the fuel mass fraction in the unburned gas, $A_{0}$ is the flow cross-section area, and $\dot{\omega}_{F}$ is the fuel reaction rate. It is well known that $s_{T}$ is sensitive to the oncoming turbulence as well as to the thermo-chemical properties of the mixture and to the flame configuration. Under increasing turbulence intensity $u^{\prime}$, with all other parameters held constant, the variation of $s_{T}$ is found to be nonlinear [1]. This behaviour, known as the bending effect, has been reviewed in-depth $[2,3]$, but it has not been explained as yet.

The present work has captured the classical bending effect (shown in Fig. 1 and discussed in Section 5.1) for the first time using Direct Numerical Simulation (DNS). In this article, we discuss the observed effect and seek an

\footnotetext{
* Corresponding author

Email addresses: gvn22@cam.ac.uk (Girish V. Nivarti), rsc10@cam.ac.uk (R. Stewart Cant)
} 
explanation for it in terms of the underlying turbulence-flame interactions as recorded in the DNS study.

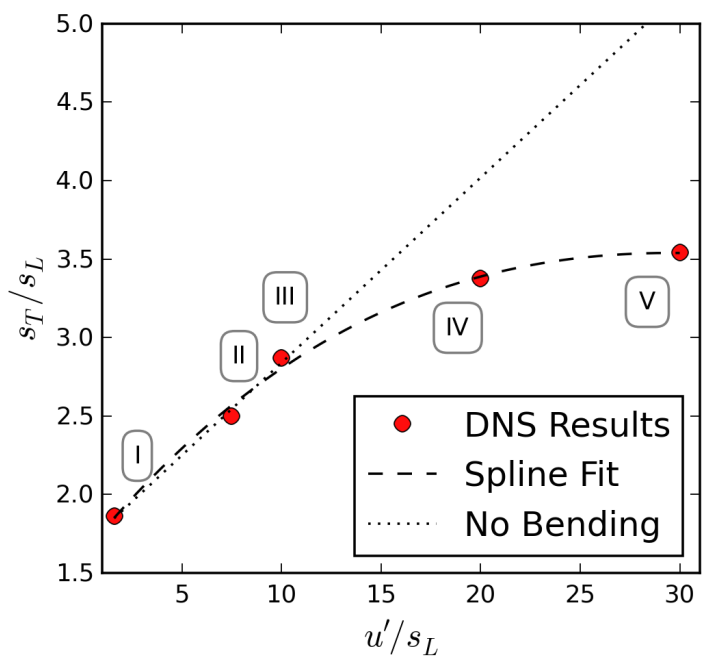

Figure 1: Calculations from the present DNS cases (I - V) plotted to show the nonlinear "bending" curve of $s_{T}\left(u^{\prime}\right)$ : turbulent burning velocity diminishes in high-intensity turbulence.

\section{Theoretical Background}

Damköhler's hypothesis [4] conjectured that, in low-intensity turbulence, $s_{T}$ increases primarily because the turbulent flow field enhances the premixed flame surface area $A_{T}$ as

$$
s_{T} / s_{L} \sim A_{T} / A_{L},
$$

where $s_{L}$ is the laminar flame consumption speed and $A_{L}$ is the laminar flame area. The underlying assumption was that $s_{L}$ remains valid locally on the flame surface in low-intensity turbulence. Subsequently, the applicability of Damköhler's hypothesis in moderate-intensity turbulence has been supported by 2D DNS [5], experimental measurements [6], and scaling analysis [7]. High-intensity turbulence - where the bending effect and its underlying processes occur - has remained elusive.

In recent years, the simultaneous advancement of laser diagnostics and supercomputing resources has opened highintensity turbulent combustion to quantitative inquiry. A variety of physical scalings that characterize the bending effect have been investigated [8] and the limits of the $s_{T}\left(u^{\prime}\right)$ curve have been explored [9]. Some experimental studies have even questioned the validity of Damköhler's hypothesis in high-intensity turbulence [10]. At the same time, large-scale DNS is beginning to provide insights [11, 12].

To date, it remains to be ascertained whether $s_{T}\left(u^{\prime}\right)$ indeed varies nonlinearly in the absence of heat losses. Given that $s_{T}\left(u^{\prime}\right)$ undergoes this bending effect, its governing mechanism has not been outlined either qualitatively or quantitatively and its consistency with the flamelet assumption is yet to be determined.

A common framework used in both experimental and computational analyses is the Flame Surface Density (FSD) approach [13]. Under the flamelet assumption, the mean flame surface-to-volume ratio $\Sigma$ captures the turbulenceflame interactions which determine $s_{T}[14]$

$$
s_{T} \sim s_{L} \int_{-\infty}^{\infty} \Sigma d \eta
$$




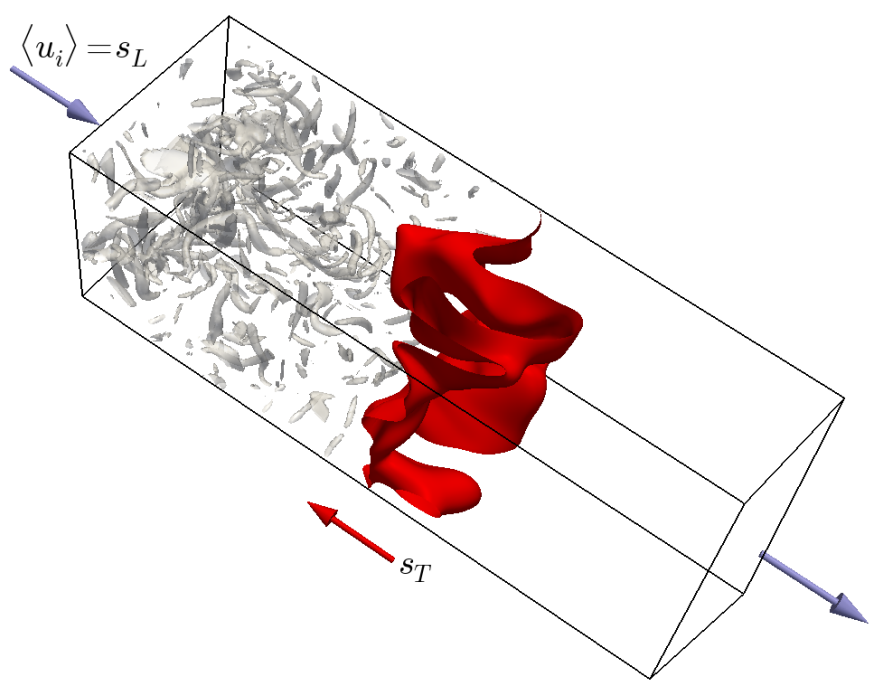

Figure 2: The 3D inflow-outflow configuration used for simulating a statistically-planar methane-air flame. In red is depicted a flame isosurface and in grey are shown contours of enstrophy.

when integrated throughout the premixed flame brush. The quantity $\Sigma \equiv\left\langle\Sigma^{\prime}\right\rangle$ is the mean of the local surface-tovolume ratio $\Sigma^{\prime}$ for a propagating flame surface and it evolves according to the transport equation [13]

$$
\frac{\partial \Sigma}{\partial t}+\nabla \cdot\left(\left\langle\mathbf{u}+s_{d} \mathbf{n}\right\rangle_{s}\right)=\langle\dot{s}\rangle_{s} \Sigma
$$

where $\mathbf{n}=-\left.[(\nabla c) /(|\nabla c|)]\right|_{c=c^{*}}$ is the local normal to the surface and $s_{d}=\left.[(\dot{\omega}+\nabla \cdot \rho \mathcal{D} \nabla c) / \rho|\nabla c|]\right|_{c=c^{*}}$ is the local surface displacement speed. All of these quantities, including the surface averaging $\langle\phi\rangle_{s} \equiv\left\langle\phi \Sigma^{\prime}\right\rangle / \Sigma$, are computed on iso-surfaces $c=c^{*}$ of the reaction progress variable $c \equiv\left(Y_{u}-Y\right) /\left(Y_{u}-Y_{b}\right)$. The generalised local FSD $|\nabla c|$, defined in the context of LES [15], is closely related to the local flame surface-to-volume ratio $\Sigma^{\prime}$. Since $\lim _{\Delta \rightarrow 0} \Sigma^{\prime}=|\nabla c|$, we use the generalised FSD to calculate $\Sigma^{\prime}$ as, in DNS, the filter size $\Delta \rightarrow 0$.

The source term $\dot{s}$ represents local flame stretch rate on the iso-surface. It is more insightful to decompose $\dot{s}$ into the separate contributions from tangential strain rate $a_{t}$ and mean curvature $h_{m}$ according to

$$
\dot{s}=a_{t}+s_{d} h_{m}
$$

where the components are given by

$$
\begin{aligned}
a_{t} & =\nabla \cdot \mathbf{u}-\mathbf{n n}: \nabla \mathbf{u}, \text { and } \\
h_{m} & =\nabla \cdot \mathbf{n} .
\end{aligned}
$$

The FSD approach links turbulent burning velocity $s_{T}$ to local events such as flame surface wrinkling, flameletmerging and intermittent quenching that might affect it (via Eq. (3)). Such events have been conjectured, in the Bunsen configuration, as likely mechanisms for the bending effect [16]. Therefore, in the present study, we use the FSD approach to seek the signatures of the bending effect. 


\section{Flame Configuration}

A statistically planar premixed flame propagating in the streamwise direction of a 3D inflow-outflow domain was simulated. An illustration of the flame propagating in a turbulent flow field is shown in Fig. 2.

The interacting turbulent flow field is prepared using a procedure akin to previous studies. First, a field of homogeneous isotropic turbulence (HIT) with intensity $u^{\prime}$ and integral length scale $\ell_{0}$ is specified in Fourier space using a model energy spectrum. The Batchelor-Townsend spectrum [17] was chosen as it represents low Reynolds number HIT in the final period of decay. The HIT field in Fourier space is then transformed to a periodic Cartesian domain where it is allowed to evolve for a short time.

The time-evolved flow field is copied multiply into the flame simulation domain. This domain has a streamwise extent given by $L_{x}=3 L_{y}=3 L_{z}=1.5 \mathrm{~cm}$ so as to avoid any flame boundary interactions. Another copy of the time-evolved flow field is convected simultaneously through the inlet at a mean axial velocity, $\left\langle u_{i}\right\rangle=s_{L}$. This inflow establishes a spatial turbulence profile upstream of the flame. In Tab. 1, key scales of the turbulent flow fields using in each simulation are listed along with inlet $u_{i}^{\prime}$ and flame leading edge $u_{l e}^{\prime}$ turbulence intensities.

A 1D laminar flame solution calculated a priori specifies the initial thermo-chemical field in the domain. The premixed methane-air laminar flame chemistry is described using a single-step Arrhenius expression formulated as

$$
\dot{\omega}=B \rho(1-c) e^{-E_{a} /(R T)},
$$

where $B=2.75 \times 10^{7} \mathrm{~m}^{3} \mathrm{~kg}^{-1} \mathrm{~s}^{-1}$ is the pre-exponential factor, $E_{a}=10^{8} \mathrm{Jkg}^{-1}$ is the activation energy and the gas constant $\mathrm{R}=287.1 \mathrm{Jkg}^{-1} \mathrm{~K}^{-1}$. The ambient pressure $P_{a}=1$ bar and the inlet temperature $T_{a}=300 \mathrm{~K}$. Other key thermo-chemical properties are reported in Tab. 2.

The present study demonstrates that the use of single-step chemistry is sufficient to capture the bending effect in stoichiometric methane-air flames. Nevertheless it is recognised that a more realistic treatment of chemistry may introduce additional effects that could influence the observed behaviour significantly. Indeed we have also carried out simulations using multi-step chemistry [18]. These simulations, however, were not extended into a full parametric study primarily due to the large computational expense involved. Moreover, despite the use of detailed chemistry, the simulated FSD terms exhibited similar variations to those observed in corresponding single-step chemistry studies. Neither did the flame display any signs of local quenching. The inclusion of a detailed chemical mechanism is, therefore, unlikely to affect drastically the conclusions of the present study.

Dimensionless regime markers (integral Reynolds $\mathrm{Re}_{0}$, Damköhler Da, and Karlovitz Ka numbers) for the five different simulations have been listed in Tab. 1. The Karlovitz number Ka is based on the Taylor micro-scale strain-rate [1]

$$
\mathrm{Ka}=\frac{u^{\prime}}{\lambda} \frac{\delta_{L}}{s_{L}} \sim\left(\frac{u^{\prime}}{s_{L}}\right)^{2} \operatorname{Re}_{0}^{-\frac{1}{2}} \sim\left(\frac{u^{\prime}}{s_{L}}\right)^{\frac{3}{2}}\left(\frac{\delta_{L}}{\ell_{0}}\right)^{\frac{1}{2}},
$$

and is used to demarcate the corrugated flamelet, thin reaction zones, and distributed burning regimes. Across all the simulations, only a single parameter (inlet turbulence intensity $u_{i}^{\prime}$ ) was varied. This is illustrated in Fig. 3 using a regime diagram. 


\begin{tabular}{lrrrrr}
\hline & I & II & \multicolumn{1}{c}{ III } & \multicolumn{1}{c}{ IV } & \multicolumn{1}{c}{$\mathrm{V}$} \\
\hline$u_{i}^{\prime} / s_{L}$ & 1.50 & 7.50 & 10.00 & 20.00 & 30.00 \\
$u_{l e}^{\prime} / s_{L}$ & 0.94 & 4.50 & 6.00 & 12.00 & 18.00 \\
$\ell_{0}(\mathrm{~mm})$ & 0.99 & 0.99 & 0.99 & 0.99 & 0.99 \\
$\lambda(\mathrm{mm})$ & 0.56 & 0.56 & 0.56 & 0.56 & 0.56 \\
$\eta(\mu \mathrm{m})$ & 71.83 & 33.18 & 28.73 & 20.31 & 16.59 \\
$\tau_{0}(\mathrm{~ms})$ & 1.59 & 0.34 & 0.25 & 0.12 & 0.08 \\
\cline { 2 - 6 } $\mathrm{Re}_{0}$ & 39.28 & 184.13 & 245.51 & 491.03 & 736.51 \\
$\mathrm{Ka}$ & 1.20 & 12.36 & 19.08 & 53.96 & 99.82 \\
$\mathrm{Da}$ & 1.71 & 0.36 & 0.27 & 0.13 & 0.09 \\
\hline
\end{tabular}

Table 1: Upstream turbulence properties and dimensionless parameters for each of the 5 simulated cases.

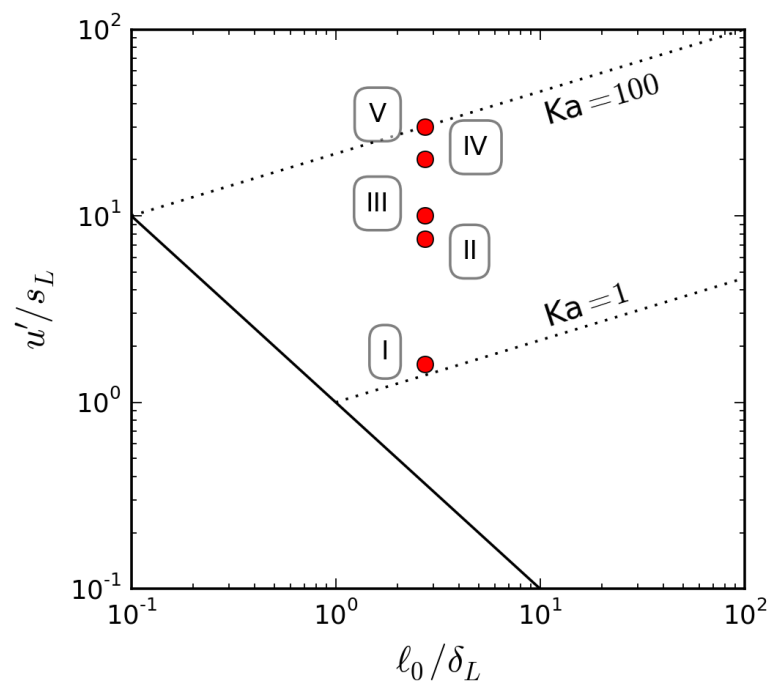

Figure 3: Turbulent combustion regimes covered by the current parametric DNS investigation. The simulations span the entire regime from corrugated to broken reaction zones.

\section{Numerical Method}

A compressible 3D formulation of the Navier-Stokes equations is solved along with thermo-chemical transport using the CFD code SENGA2 [19]. In this code, a $10^{\text {th }}$-order centred finite difference operator evaluates spatial derivatives within the domain. The operator reduces to $4^{\text {th }}$ order at non-periodic boundaries. In the streamwise direction, inflow-outflow boundaries are specified using the Navier-Stokes characteristic boundary conditions [20]. Transverse directions are specified with periodic boundaries. Time-marching is conducted using a $4^{\text {th }}$-order explicit Runge-Kutta method along with adaptive time-stepping based on error control [21]. Due to the simple chemistry, a relatively coarse mesh can be used which nevertheless resolves the turbulence microscales. A fixed mesh size $N=288 \times 96 \times 96$ with a uniform spacing $\Delta x=52.3 \mu m \sim \mathcal{O}(\eta)$ is used across all cases. The DARWIN (Intel Sandy Bridge E5-2670) and ARCHER (Cray XC30) supercomputers were used for the computations; a typical simulation used $\sim 2.5 \times 10^{4}$ core-hours. 


\begin{tabular}{lr}
\hline Property & Value \\
\hline Heat release parameter, $\tau$ & 6.33 \\
Lewis number, Le & 1.00 \\
Prandtl Number, Pr & 0.70 \\
Schmidt Number, Sc & 0.70 \\
Laminar flame speed, $s_{L}\left(\mathrm{~ms}^{-1}\right)$ & 0.39 \\
Laminar flame thickness, $\delta_{L}(\mathrm{~mm})$ & 0.36 \\
\hline
\end{tabular}

Table 2: Thermo-chemical parameters, dimensionless groups and unstrained laminar flame properties.

\section{Computational Results}

\subsection{Observation of Bending}

In Fig. 1, the bending effect of the $s_{T}\left(u^{\prime}\right)$ curve has been presented as captured by the present DNS. Each dot corresponds to $s_{T}$ calculated for a unique simulation with specified $u^{\prime}$. The calculations of $s_{T}$ have been done at a time corresponding to 4 eddy turn-over times $t=4 \tau_{0}$ for each case. Turbulent flow kinematics reached statistical convergence by this time and, subsequently, $s_{T}$ exhibited only minor fluctuations about the plotted value. The deviation from linearity of the $s_{T}\left(u^{\prime}\right)$ curve is fully captured within cases II - V with $u^{\prime} \geq 7.5 s_{L}$.

In the following sections, we analyse cases II - V and evaluate the FSD source term components (RHS in Eq. (4)) as recorded in these cases. Where the analysis is restricted to a single flame isosurface, the contour $c^{*}=0.8$ is chosen as this corresponds to the peak fuel reaction rate contour for an unstrained laminar flame described by the present single-step chemistry. Statistics of all quantities $\phi$ have been area-weighted $\mathrm{P}(\phi)=\phi \Sigma^{\prime} /\left\langle\Sigma^{\prime}\right\rangle$ where the angle brackets denote averaging over transverse directions and normalized $\widehat{\phi}$ such that $\int_{-\infty}^{\infty} \mathrm{P}(\widehat{\phi}) d \widehat{\phi}=1$.

\subsection{Tangential Strain Rate}

In Fig. 4a, the distribution of tangential strain rate $a_{t}$ on the $c^{*}=0.8$ isosurface is shown. The strain rate has been normalized using the Taylor scale strain rate $u^{\prime} / \lambda$. Turbulent straining of the flame surface occurs close to the Taylor length scale $\lambda$ as suggested previously [1, 14]. All the cases exhibit an extensional mean surface strain $\left\langle a_{t}\right\rangle_{s}>0$ consistent with theory on turbulent transport of material surfaces [13]. Further, the distribution shapes throughout all cases are similar to those observed previously in lower intensities of turbulence [14].

\subsection{Mean Curvatures}

The variation of local mean curvature $h_{m}$ of the $c^{*}=0.8$ isosurface is shown in Fig. 4b for cases II - V. For consistency with strain rate scaling, the Taylor length scale $\lambda$ is chosen here as the scaling parameter. For all cases, the surface average of mean curvature $\left\langle h_{m} \lambda\right\rangle_{s} \approx 0$, conforming with previous observations [14]. The progressively increasing likelihood of negative mean curvatures with increasing $u^{\prime}$ is evident.

\subsection{Displacement Speed}

Fig. 4c shows profiles of displacement speed $s_{d}$ for cases II - V at the isosurface $c^{*}=0.8$. Under higher levels of turbulence intensity $u^{\prime}$, the variance of $\left.s_{d}\right|_{c=0.8}$ increases due to increased turbulent fluctuations experienced by the reaction layer. In accordance, negative $s_{d}$ becomes increasingly common in higher intensities of turbulence. This is in line with previous 2D DNS studies [22]. The surface mean $\left\langle s_{d}\right\rangle_{s}$ reduces gradually up to case IV where $u^{\prime} / s_{L}=20$. In the highest intensity case (V), however, a further reduction is observed indicating a greater role played by turbulent 


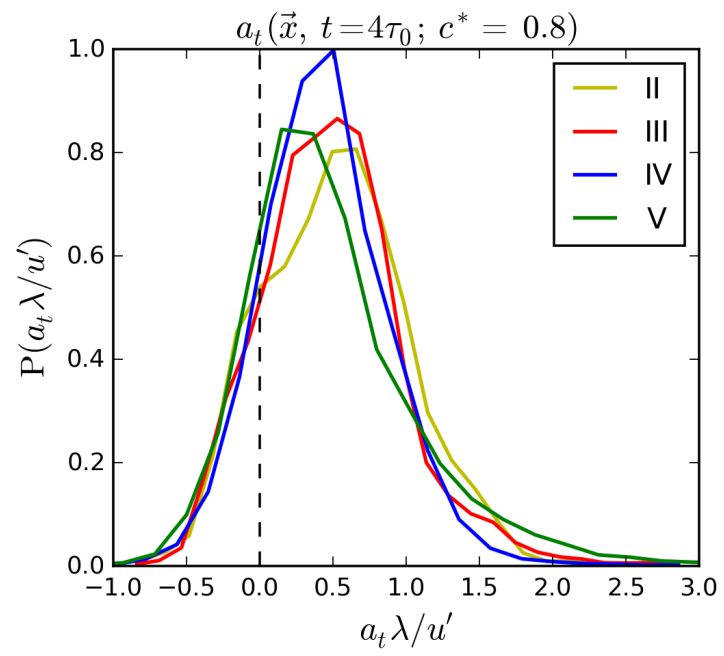

(a) Normalized tangential strain rate $\left(a_{t}\right)$

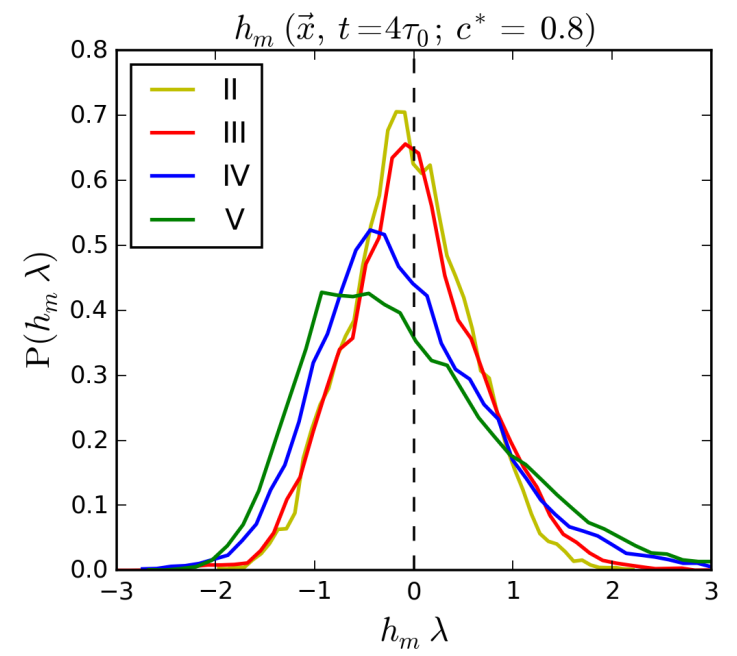

(b) Normalized mean curvature $\left(h_{m}\right)$

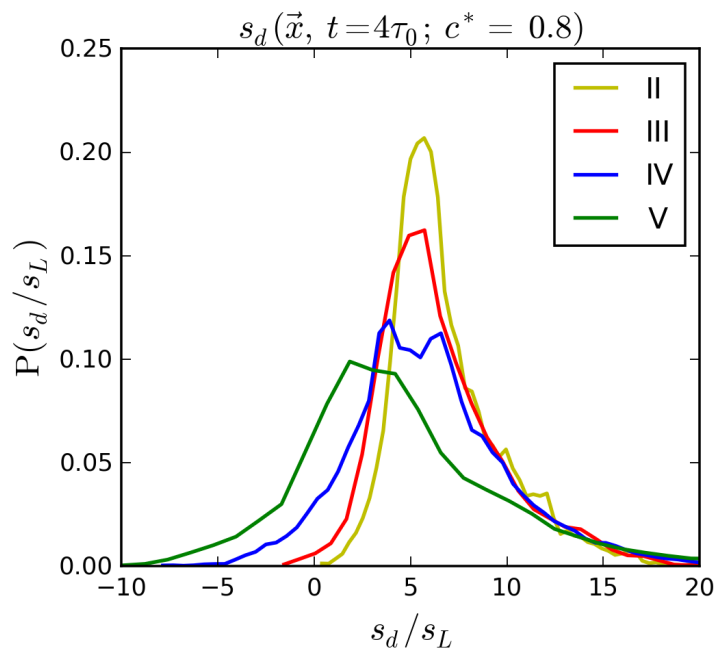

(c) Displacement speed $\left(s_{d}\right)$

Figure 4: Distribution of FSD source-terms over the reaction layer $c=0.8$ in moderate and high intensity cases (II - V). 


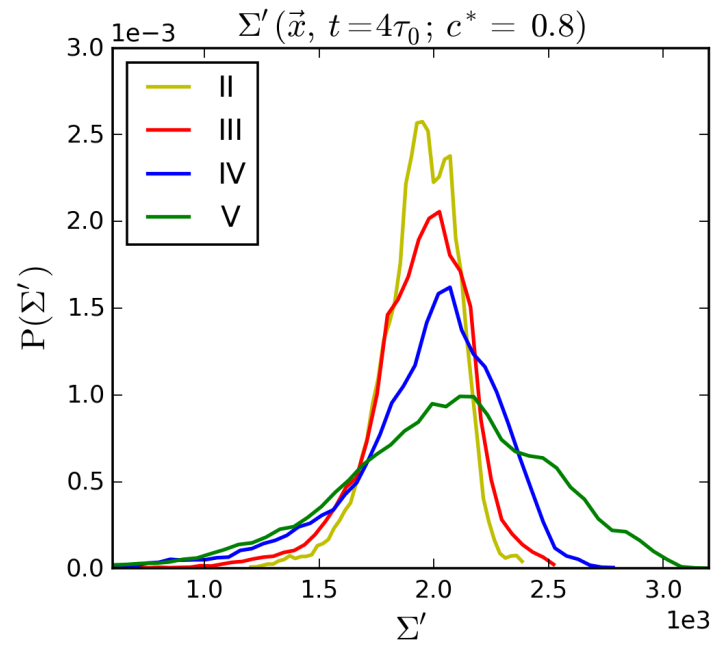

(a) Local FSD distribution $\left.\Sigma^{\prime}\right|_{c^{*}=0.8}$

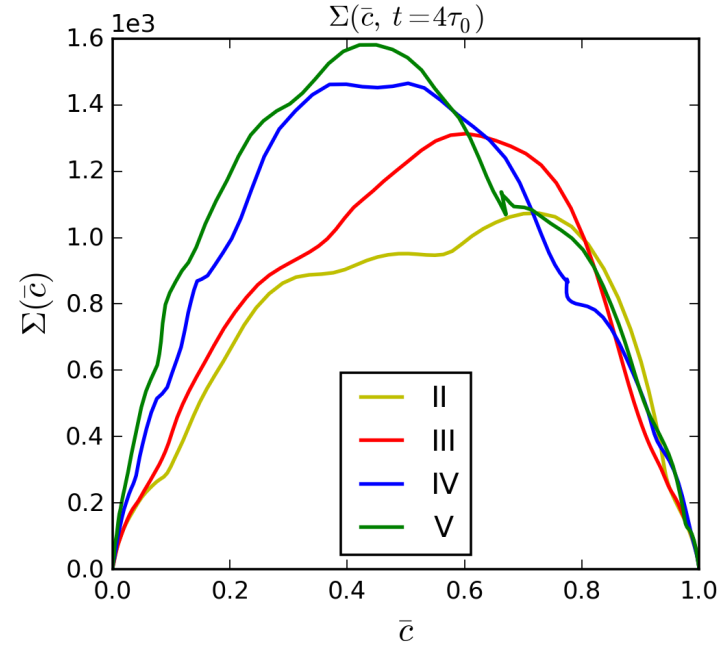

(b) Mean FSD $\Sigma(\bar{c})$ across the mean flame brush

Figure 5: FSD profiles for moderate to high turbulence intensity.

fluctuations. Further investigation, beyond the scope of the present study, will help understand the implications of this observation.

\subsection{Flame Surface Density}

The distribution of local FSD $\Sigma^{\prime}$ over the $c^{*}=0.8$ isosurface is shown in Fig. 5a. The increased variance of $\left.\Sigma^{\prime}\right|_{c=c^{*}}$ with increasing $u^{\prime}$ is to be expected $[1,13,16]$ due to increased turbulent fluctuations. The average of $\left.\Sigma^{\prime}\right|_{c=c^{*}}$ over the isosurface, however, increases only marginally across the cases. The effect of $u^{\prime}$ on $\Sigma^{\prime}$ across the turbulent flame brush is visible in Fig. 5b. The mean FSD $\Sigma(\bar{c}) \equiv\left\langle\Sigma^{\prime} \mid \bar{c}=c^{*}\right\rangle$ increases with $u^{\prime}$, but the changes are restricted mainly to the pre-heat zone $\bar{c}<0.6$. The mean flame brush tail $\bar{c}^{*}>0.6$ does not experience marked differences in $\Sigma$ under varying turbulence intensity $u^{\prime}$. The peak mean FSD $\Sigma_{\max }$ reflects this behaviour - higher peaks are achieved in higher intensities of turbulence, and these peaks drift towards the leading edge of the flame with increasing $u^{\prime}$.

Fig. 6 shows images of the turbulent premixed flame brush for the high-intensity turbulence cases coloured by FSD $\Sigma^{\prime}$. Each slice represents the case at $t=4 \tau_{0}$ and is taken from the $3 \mathrm{D}$ domain at $y=L_{y} / 4$. The images in Fig. 6 illustrate a gradual progression - while under $u^{\prime} / s_{L}=10$, laminar flamelet structure is retained throughout the flame brush, the successive cases show a disturbance in flamelet structure of the preheat zone. The reaction layers, however, retain largely sheet-like parallel surfaces despite minor local disruptions such as those visible in case $\mathrm{V}$ where $u^{\prime} / s_{L}=30$. This observation is consistent with Fig. 5b and with previous theory on the thin reaction zones regime [7]. Also worth noting is that while the peak local FSD $\Sigma_{\text {max }}^{\prime}$ appears to increase with $u^{\prime}$, at the same time it appears to be confined increasingly to highly localised regions.

\section{Discussion and the Mechanism of Bending}

The turbulent premixed flame surface area $A_{T}$ calculated as the conditionally integrated FSD

$$
A_{T}=\int_{-\infty}^{\infty} \Sigma^{\prime} \delta\left(c-c^{*}\right) d V
$$




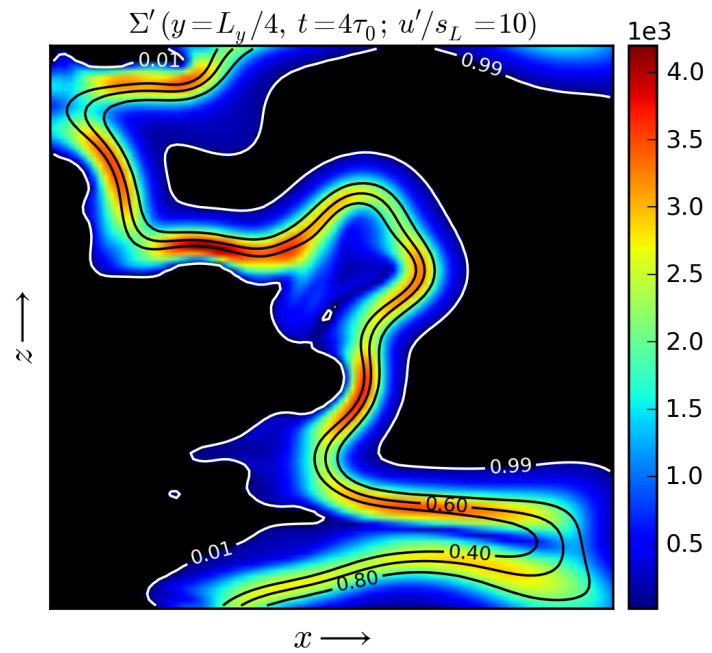

(a) Case III: $u^{\prime} / s_{L}=10$

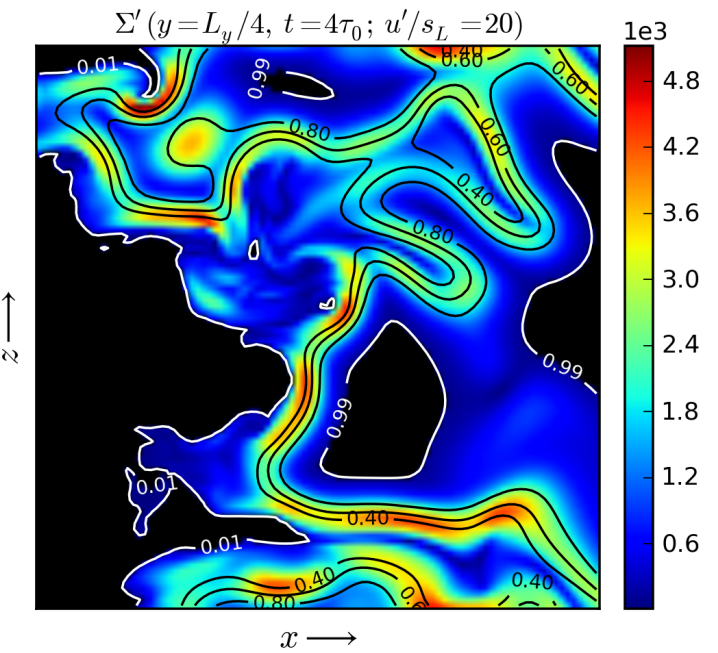

(b) Case IV: $u^{\prime} / s_{L}=20$

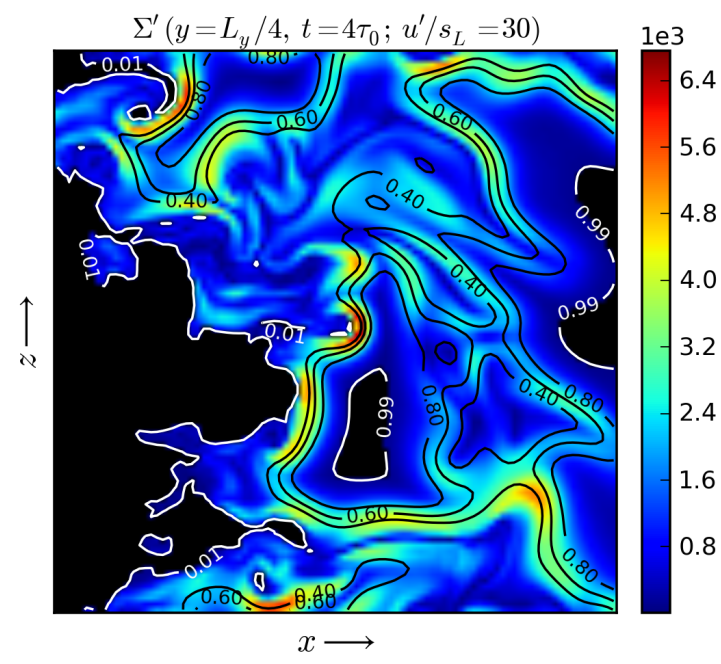

(c) Case V: $u^{\prime} / s_{L}=30$

Figure 6: Flame Surface Density $\Sigma^{\prime}$ for high turbulence intensity (cases III, IV, V) showing gradually increasing effects of in the flame brush. 
in the reaction layer $\left(c^{*}=0.8\right)$ is shown alongside the bending curve in Fig. 7. The close match between $s_{T} / s_{L}$ (red line) and $A_{T} / A_{L}$ (blue line) confirms the validity of Damköhler's hypothesis under conditions that cause the bending effect to occur.

The turbulent flame brush volume $V_{T}$ normalized with the laminar flame volume $V_{L}=A_{L} \delta_{L}$ increases monotonically with $u^{\prime}$ (green line in Fig. 7). Hence, the mean FSD $\Sigma(\bar{c})$ and peak FSD $\Sigma_{\text {max }}^{\prime}$ (discussed in Sec. 5.5) as well as flame brush volume $V_{T}$ increase monotonically with turbulence intensity $u^{\prime}$. Yet the respective mean turbulent burning velocities $s_{T}$ follow a bounded non-linear curve. This is investigated further using FSD source terms integrated

$$
\widehat{a}=\frac{1}{V} \int_{-\infty}^{\infty} a_{t} d V, \text { and } \widehat{h}=\frac{1}{V} \int_{-\infty}^{\infty} s_{d} h_{m} d V
$$

over the entire domain of volume $V$. These quantities are shown alongside the bending curve in Fig. 7 using dashed

lines (upright triangles: $\widehat{a}$ and inverted triangles: $\widehat{h}$ ). The volume-integrated tangential strain rate $\widehat{a}$ is positive for all $u^{\prime} / s_{L}$ values considered and hence acts to increase $A_{T}$. Conversely, the volume-integrated curvature term $\widehat{h}$ is negative for all $u^{\prime} / s_{L}$ values and acts to decrease $A_{T}$. Under high-intensity turbulence the effect of $\widehat{h}$ appears to dominate, reducing $A_{T}$ below its value extrapolated linearly from low $u^{\prime}$. The (im)balance between $\widehat{a}$ and $\widehat{h}$ appears to play a critical role in the bounded growth of $A_{T}$ which, through Damköhler's hypothesis, causes the inhibited growth of $s_{T}$ at high $u^{\prime}$. The shift in the balance of $\widehat{a}$ and $\widehat{h}$ occurs gradually with increasing $u^{\prime}$, and there are no sudden transitions.

\subsection{Phenomenological Explanation}

The analysis confirms that the observed bending effect occurs as a result of inhibited growth of the flame surface area in high intensity turbulence. The effect is determined quantitatively by surface strain and curvature terms. Qualitatively, the bending effect may be understood in terms of reduced efficacy of turbulent eddies to strain the flame as turbulence intensity increases. While the smaller eddies of intense turbulence continue to be efficient at creating curvature in the flame, these are not strong enough to strain it. Further, these strongly curved regions of the flame surface tend to have negative curvature, contributing to a negative stretch rate that tends to decrease flame surface area. The preference for negative over positive curvatures is a topic of further study.

\section{Conclusions}

A bending effect is captured by a parametric 3D DNS study of statistically planar methane-air flames subject to increasing turbulence intensity $u^{\prime}$. Damköhler's hypothesis retains its validity throughout this bending curve. The turbulent flame surface area $A_{T}$ determines the burning velocity $s_{T}$ even for the highest intensity simulation $\left(u^{\prime} / s_{L}=30\right)$. Leading order FSD source terms - namely, tangential strain $a_{t}$ and mean curvature $h_{m}$ - are able to capture the observed nonlinear variation of $A_{T}$. The reaction layer tends to be preserved and it appears that local flame quenching does not govern bending. Future simulations of longer duration and with multi-step chemistry will lend greater insight into the effects observed here and other effects that may influence bending.

\section{Acknowledgements}

The authors acknowledge the support of the Cambridge High Performance Computing Cluster as well as ARCHER UK National Supercomputing Service. GVN would like to thank Giles Shaw and Dr. Artan Sheshmani for helpful 


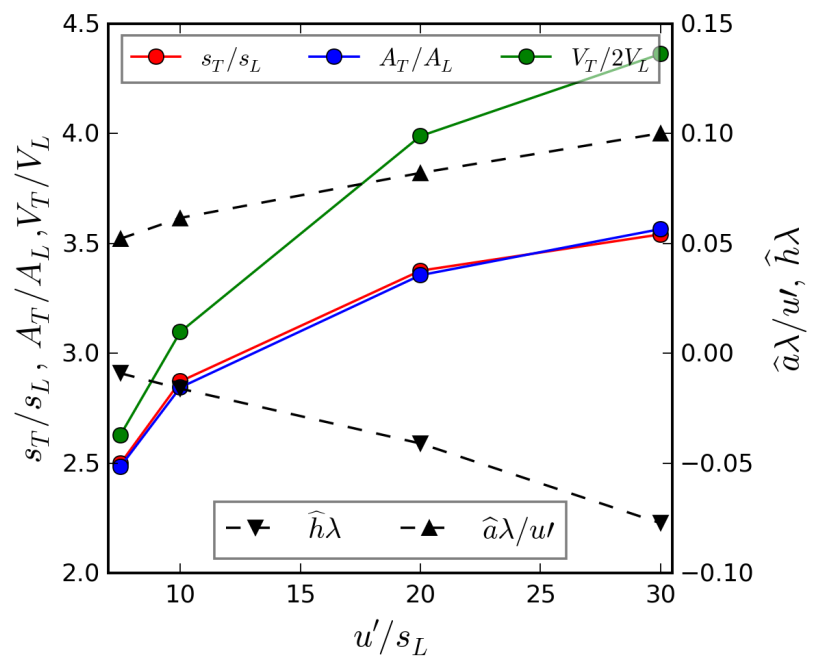

Figure 7: The bending curve $s_{T}\left(u^{\prime}\right)$ alongside integrated FSD terms as well as the flame brush volume $V_{T}$. Solid lines follow the left hand side axis whereas dashed lines follow the right. The gradual shift in balance between integrated turbulent straining and curvature terms with turbulence intensity is apparent.

mathematical discussions.

[1] D. Bradley, Symposium (International) on Combustion (1) (1992) 247-262.

[2] A. Lipatnikov, J. Chomiak, Progress in Energy and Combustion Science (1) (2002) 1-74.

[3] J. F. Driscoll, Progress in Energy and Combustion Science (1) (2008) 91-134.

[4] G. Damköhler, Zeitschrift für Elektrochemie und angewandte physikalische Chemie (11) (1940) 601-652.

[5] C. Meneveau, T. J. Poinsot, Combustion and Flame (1991) 311-332.

[6] B. Bedat, R. K. Cheng, Combustion and Flame (August 1994) (1995) 485-494.

[7] N. Peters, Journal of Fluid Mechanics (1999) 107-132.

[8] S. S. Shy, W. J. Lin, J. C. Wei, Proceedings of the Royal Society A: Mathematical, Physical and Engineering Sciences (2000) (2000) 1997-2019.

[9] D. Bradley, M. Lawes, K. Liu, M. S. Mansour, Proceedings of the Combustion Institute (1) (2013) 1519-1526.

[10] F. T. Yuen, O. L. Gülder, Proceedings of the Combustion Institute (1) (2013) 1393-1400.

[11] A. J. Aspden, M. S. Day, J. B. Bell, Journal of Fluid Mechanics (2011) 287-320.

[12] A. Y. Poludnenko, E. Oran, Combustion and Flame (2) (2011) 301-326.

[13] S. B. Pope, International Journal of Engineering Science (5) (1988) 445-469.

[14] K. N. C. Bray, R. S. Cant, Proceedings of the Royal Society A: Mathematical, Physical and Engineering Sciences (1890) (1991) 217-240. 
[15] M. Boger, D. Veynante, H. Boughanem, A. Trouvé, Symposium (International) on Combustion (1) (1998) 917-925.

[16] S. A. Filatyev, J. F. Driscoll, C. D. Carter, J. M. Donbar, Combustion and Flame (1-2) (2005) 1-21.

[17] G. K. Batchelor, A. A. Townsend, Proceedings of the Royal Society A: Mathematical, Physical and Engineering Sciences (November) (1948) 527-543.

[18] G. V. Nivarti, R. S. Cant, $25^{\text {th }}$ international conference for dynamics of explosions and reactive systems, 2015.

[19] R. S. Cant, Tech. Rep. CUED-THERMO-2012/04 (2), Cambridge University Engineering Department (2013).

[20] J. C. Sutherland, C. A. Kennedy, Journal of Computational Physics (2) (2003) 502-524.

[21] C. A. Kennedy, M. H. Carpenter, Applied Numerical Mathematics (1-2) (2003) 139-181.

[22] N. Peters, P. Terhoeven, J. H. Chen, T. Echekki, Symposium (International) on Combustion (1) (1998) 833-839. 